\title{
Predicting split decisions in MPEG-2 to HEVC video transcoding
}

\author{
Tamer Shanableh $^{1} \mathbb{D} \cdot$ Mahitab Hassan $^{2}$
}

Received: 19 January 2020 / Accepted: 13 May 2020 / Published online: 25 May 2020

(c) Springer Nature Switzerland AG 2020

\begin{abstract}
This paper proposes learning-based approaches for transcoding videos compressed using the Moving Picture Experts Group 2 format into the High Efficiency Video Coding (HEVC) format. In the training mode of the transcoder, mappings between extracted features and split decisions are calculated. While in the transcoding mode, the split decisions of Coding Units of the HEVC video are predicted. Two formulations are proposed for the prediction of split decisions based on multi model and multi-tier solutions. In the former solution, multi models are generated based on the total number of split flags in a coding unit. While in the latter solution, split decisions are modelled at three different coding depths. The proposed solutions are evaluated in terms of excessive bitrate, drop in PSNR, classification accuracy, model generation time and transcoding speedup. It is shown that the multi-tier solution maintains the rate-distortion behaviour of full re-encoding at the expense of lower gain in transcoding speedup. In comparison to existing work, it is shown that the proposed solutions offer a significant enhancement in terms of rate-distortion performance and classification accuracy.
\end{abstract}

Keywords Video coding $\cdot$ Video transcoding $\cdot \mathrm{HEVC} \cdot$ Machine learning

\section{Introduction}

One of the main objectives of the High Efficiency Video Coding (HEVC) is to provide a significant rate-distortion improvement in comparison to H.264/AVC. Such an improvement paves the way for new applications requiring ultra-high definition resolutions [1].

Heterogeneous video transcoding can be applied to convert existing videos compressed with popular standards such as MPEG-2 and H.264/AVC into HEVC. The term heterogeneous is used to indicate facilitating interoperability between different video coding standards. One of the earliest work on heterogeneous video transcoding was reported by the author in [2,3] for transcoding between different video formats.

To date, not much work has been reported for HEVC heterogeneous video transcoding. Nonetheless, a number of video transcoders are reported for transcoding between MPEG-2 and H.264/AVC on one side and HEVC on the other side. Noteworthy are the heterogeneous HEVC transcoding solutions that are based on content modelling for predicting the coding depth of HEVC CUs.

For example, in [4] it was proposed to extract features from the incoming H.264/AVC coded blocks and compare their values against adaptive thresholds to decide on the outgoing HEVC CU partitioning. These incoming features are based on Motion Vector (MV) statistics, number of DCT coefficients and energy of DCT coefficients. The adaptive thresholds are computed from the first $\mathrm{K} \mathrm{H.264/AVC}$ frames and corresponding outgoing HEVC CUs, where $\mathrm{K}$ is typically set to 25 frames.

Another approach for H.264/AVC to HEVC transcoding is based on Linear Discriminant Functions (LDFs). Features are extracted from the incoming H.264/AVC video and mapped to split or no split decisions of the outgoing HEVC CUs. Again, the weights of the LDFs are computed from the first $\mathrm{K}$ incoming frames as in the previous approach. Once computed, LDFs are used to classify outgoing CUs

Tamer Shanableh, tshanableh@aus.edu |'Department of Computer Science and Engineering, American University of Sharjah, Sharjah, UAE. ${ }^{2}$ IBM Cloud, Dubai, UAE. 
between split or no split [5]. One exception applies to incoming blocks with a high MV variance, such blocks are automatically classified as split [4].

Parallel processing has been used to speedup the H.264/ AVC to HEVC transcoding processing. The transcoder made use of incoming coding information for further speedup as well. It was reported that $720 p$ resolution video can be transcoded in real time [6].

Other transcoding solutions exist, for instance, in [7] it was proposed to segment incoming H.264/AVC frames into three regions based on coding complexity. After that, the coding structure of an outgoing $\mathrm{CU}$ is determined based on the incoming region type and MVs. A MV clustering techniques of incoming MVs is also proposed for reducing the complexity of H.264/AVC to HEVC video transcoding [8].

On the other hand, since the MPEG-2 video content is widely available, a MPEG-2 to HEVC transcoder is proposed in [9]. The transcoding results are attractive in terms of excessive bitrate and computational complexity. However, the learning approach used is limited in that it predicts the coding depth of the first sub CU in a $64 \times 64$ coding unit. The predicted depth is replicated to the rest of the sub CUs in the same CU.

With the co-existence of many video coding standards, codec interoperability tools like video transcoders are becoming important. With the latest ITU-T-ISO/IEC HEVC codec, a need has emerged for transcoding legacy formats into HEVC. In particular, the MPEG-2 video content, which is used in TV services, digital broadcast and DVDs is plentiful. It is well-known that the MPEG-2 compression efficiency is not as good as that offered by HEVC. One approach to make use of existing MPEG-2 content whilst reducing its bitrate and file size is to transcode such videos into the efficient HEVC video codec.

The main object of this work is to propose a MPEG-2 to HEVC transcoder with an efficient learning approach to predict all split decisions of an outgoing HEVC CU. The proposed solutions are based on generating multi split decision models and three tier classification models. For that purpose, we represent the split decisions of a $64 \times 64$ CU using 21 binary digits. Each of the split decisions of a $\mathrm{CU}$ are classified separately using the multi model classification approach. Whereas in the three tier model, the split decisions are grouped according to one of the three coding depths and consequently, only three classification models are generated.

Both classification approaches are integrated in a transcoding system that uses the first $\mathrm{K}$ input and output frames for model generation. Therefore, the main contribution of this manuscript is to predict the outgoing split decisions by modelling the relationship between the incoming MPEG-2 coding parameters and the outgoing split decisions of CUs. The proposed transcoding system shall maintain the video quality in comparison to the case of full HEVC re-encoding, yet at the same time, speedup the video conversion processes.

Such a transcoding approach is needed since existing video content coded with MPEG-2 format is abundant as it is used in high definition and standard format TV and transmission systems. By transcoding from MPEG-2 to HEVC, existing MPEG-2 content can be made use of with HEVC decoders, thus, achieving compatibility with older formats. Additionally, by transcoding into HEVC format, the MPEG-2 video files can be greatly reduced in size without sacrificing its quality as shown in this paper.

The rest of the paper is organized as follows. Section 2 present a literature review on HEVC transcoding. Section 3, introduces the overall transcoding architecture, Sect. 4 introduces the proposed multi model and three-tier classification solutions, Sect. 5 presents the experimental results and Sect. 6 concludes the paper.

\section{Literature review}

In the literature, most of the HEVC transcoding work is reported for H.264 into HEVC transcoding and HEVC transrating. Nonetheless, a number of interesting transcoding solutions are reported for HEVC into non-ISO standardized coder are reported. For instance, in [10] a HEVC to Audio Visual Standard version 2 (AVS2) transcoder is proposed which decodes the incoming video in multi-stages to make use of HEVC information in the transcoding process. The authors reported speedup in the range of $11 \times$ to $17 x$ over AVS2 reference software. Likewise, [11] reported a HEVC into Video Predictor version 9 (VP9) transcoder with a $60 \%$ reduction in complexity at the expense of acceptable Rate-Distortion (R-D) penalties. On the other hand, HEVC translating is reported in [12] and [13]. The fastest solution in [12] achieved a complexity reduction of $82 \%$ with a bitrate penalty up to $3 \%$. The transcoder in [13] is based on the decoder-encoder cascade with statistical analysis to leverage CU and Prediction Unit (PU) structures from the incoming HEVC video.

It is worth mentioning that the majority of reported HEVC transcoders is related to H.264 to HEVC transcoding for both intra only and intra/inter modes. For instance, [14] proposed fast intra H.264 to HEVC transcoder by leveraging the incoming DCT coefficients and intra predictions to predict the coding depth in HEVC CUs. The transcoder is 1.7-2.5 times faster than ordinary HEVC encoding with a bitrate penalty up to $3 \%$. The work in [15] proposed a similar transcoder that utilizes Bayesian classifiers to speedup the transcoding process. Additionally, inter-frame H.264 to HEVC transcoder are reported. In [16] a low complexity 
transcoder is proposed which makes use of machine learning and parallel processing. A parallel algorithm is proposed to make use of a multi-core CPU and GPU. In [17] a transcoding algorithm is proposed that makes use of intra and inter coding information and MVs in $\mathrm{H} .264$ to accelerate the re-encoding in HEVC. In [18], incoming H.264 blocks are fused according to a motion similarity criterion. The map is used to help in constructing the quadtree of HEVC coded frames. H.264 MVs are used as a starting point for motion estimation in HEVC as well. Time savings of $63 \%$ are reported with bitrate penalty of $1.4 \%$.

In this work we complement the existing literature by proposing a MPEG-2 to HEVC transcoding solution.

\section{Transcoding architecture}

A HEVC video frame contains equal size coding units referred to as CUs. A typical size of a CU is $64 \times 64$ pixels in the luma part. A CU can be divided into smaller blocks referred to as sub-CUs. The sizes of such sub-CUs can be $32 \times 32,16 \times 16$ or $8 \times 8$. Sub-CUs can be recursively divided resulting in a quadtree structure. An example of which is shown in Fig. 1. The quadtree can have a maximum depth of three, which corresponds to a sub-CU size of $8 \times 8$ pixels. The final quadtree structure of a CU is computed using a brute-force method that considers all possible splitting arrangements using R-D optimization. The optimization takes into account motion estimation and compensation which makes it the most complex task of the encoder. Once the quadtree is structure is computed, PUs are decided upon for each sub-CU.

A sub-CU can remain as is or be further divided into 2 or 4 PUs with symmetric shapes for intra coding and symmetric/asymmetric shapes for inter coding. For the

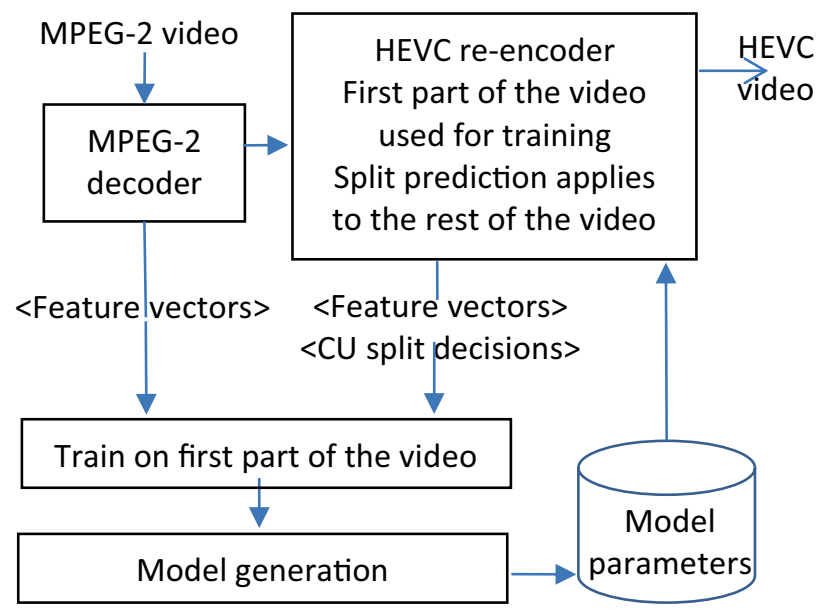

Fig. 1 Overall MPEG-2 to HEVC block diagram transformation and quantization of prediction residuals, each sub-CU is recursively partitioned into what is known as Transform Units (TUs).

Since the computation of the CU's quadtree is the most complex, the proposed transcoding system focuses on predicting the $\mathrm{CU}$ splits that result in the final quadtree.

The overall block diagram of the proposed transcoder is shown in Fig. 1. The first part of the decoded video is used for model generation. Once generated, the model parameters are used in the HEVC re-encoder to predict the quadtree structure of coding units. More specifically; to predict HEVC split decisions, the transcoding system needs to operates in two modes; a training mode and a transcoding mode as illustrated in Fig. 2 . In the training mode, which contains an MPEG-2 decoder followed by a full HEVC encoder, the first $K$ frames of the incoming MPEG-2 and the corresponding outgoing HEVC videos are used for model generation. During this mode, feature

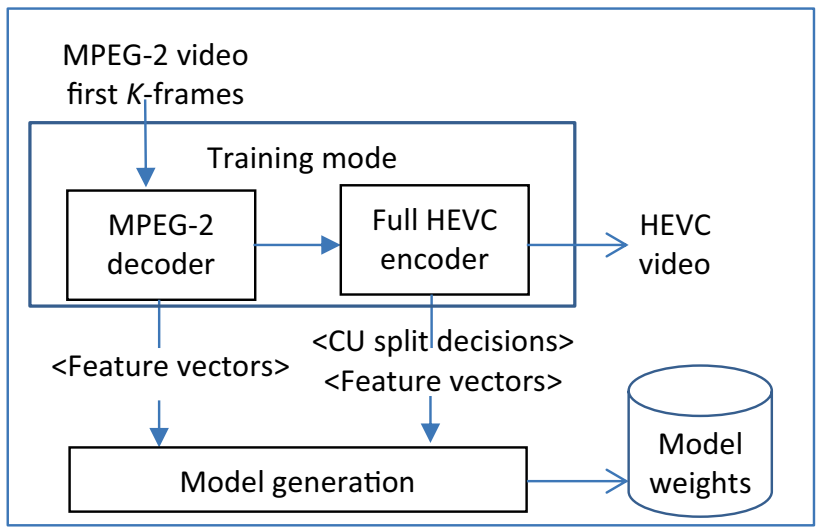

(a)

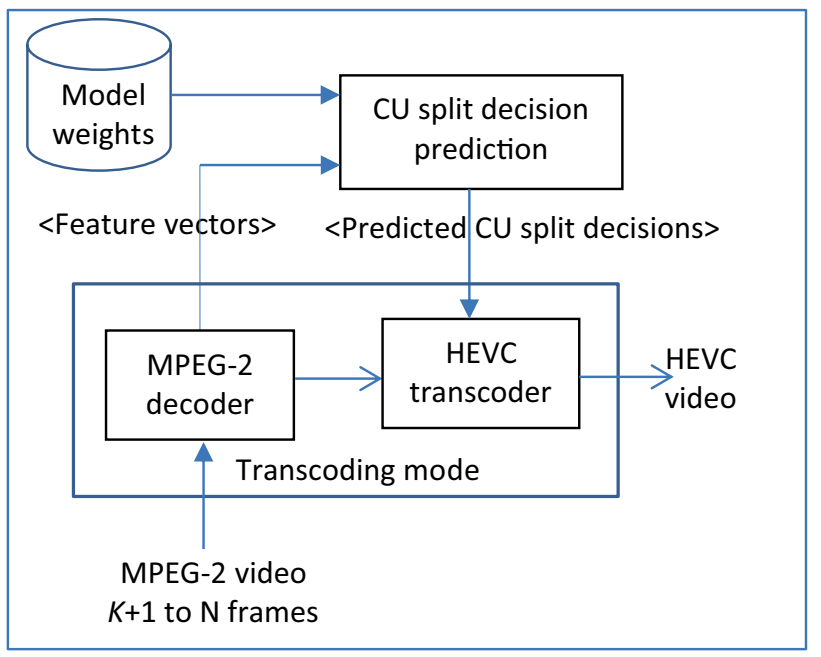

(b)

Fig. 2 MPEG-2 to HEVC transcoding architecture a Training phase, b Transcoding phase 
vectors are extracted from the incoming bit stream and the decoded video is re-encoded using HEVC. During the re-encoding, the split decisions of each CU are stored. Having collected the feature vectors and corresponding split decisions, the training mode computes a mapping between the two and generates model weights that can be used in the transcoding mode.

On the other hand, in the transcoding mode, the feature vectors of the incoming MBs together with the previously generated model weights are used to predict the split decisions of the outgoing CUs; hence, greatly simplifying the operations of the HEVC coder.

The feature vectors extracted from the MPEG-2 video are based on MVs, coding information and texture variance. The exact features are listed in Table 1.

In a $4 \times 4$ square of MBs, which corresponds to a $64 \times 64$ $\mathrm{CU}$, the following feature variables are used: the variance of the $16 \mathrm{MVs}$ in both the $x$ and $y$ directions, raw MV values, $16 \mathrm{MB}$ types, 16 coded block patterns for each MB, texture variance of each of the $16 \mathrm{MBs}$. In addition to that, MBs are arranged into $2 \times 2$ squares pertaining to $32 \times 32$ sub-CUs and the corresponding MV variances are computed and added to the feature vector.

We propose a solution for predicting the whole coding tree unit of a given $64 \times 64 \mathrm{CU}$, therefore, the coding depth of each sub CU is predicted by the transcoder. In the next section, we formulate the solution as a multi model classification problem.

\section{Proposed classification solutions}

In the following sub sections, we propose two solutions for the classification of split decisions. The first solution uses feature variables from MPEG-2 for the prediction of all split decisions of the corresponding HEVC CUs. The total number of split decisions is 21 as explained in the next section. Therefore, 21 models are generated in this classification solution. The rational behind this approach is

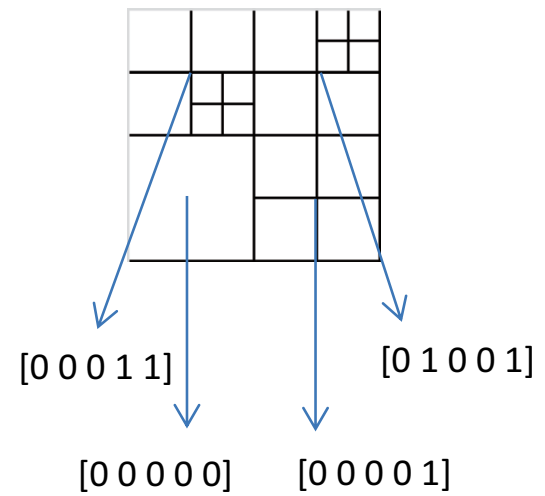

Fig. 3 Representation of split decisions

that the feature variables pertaining to $4 \times 4$ MPEG- 2 MBs correspond to one HEVC CU, hence the quadtree structure of the later can be predicted based on the extracted feature variables. We will refer to this solution as multi-model classification.

Additionally, we propose another classification solution that takes into account the three levels of splitting a CU. Considering that a typical CU has a size of $64 \times 64$ pixels, three split levels can be applied to generate sub-CUs with sizes of $32 \times 32,16 \times 16$ and $8 \times 8$. As such, the feature variables extracted for this task are applied to different numbers of MPEG-2 MBs pertaining to the sizes of the largest $\mathrm{CU}$ and sub-CUs. More specifically, three models are generated pertaining to the three split levels, where each model has it is own feature matrix. We will refer to his solution as three tier classification solution.

\subsection{Multi model classification solution}

In this proposed solution, we predict all the split decisions of a CU. To achieve this task, the split decisions of a CU in this work are represented using a sequence of $1 \mathrm{~s}$ and $0 \mathrm{~s}$ as illustrated in Fig. 3.

Table 1 Description of MPEG-2 feature variables

\begin{tabular}{l}
\hline Feature variables \\
\hline Incoming MBs are arranged in $4 \times 4$ blocks corresponding to the size of a CU. The variance of the MVs is computed \\
Raw MVs values $(x, y)$ of $4 \times 4 \mathrm{MBs}$ \\
Incoming MBs are arranged in $2 \times 2$ blocks corresponding to the size of a CU at depth 1. The variance of the MVs of each $2 \times 2$ \\
block is computed \\
MB types in the $4 \times 4$ block of MBs \\
MB Coded Block Patterns $(\mathrm{CBP})$ in the $4 \times 4$ block of MBs \\
Number of coding bits per MB of $4 \times 4 \mathrm{MBs}$ \\
Texture variances in the $4 \times 4$ block of MBs
\end{tabular}


For instance, the top left $32 \times 32 \mathrm{CU}$ of the $64 \times 64 \mathrm{CU}$ in

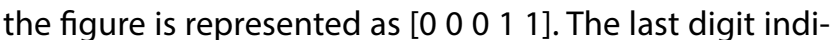
cates whether or not the $32 \times 32 \mathrm{CU}$ is split. In this example, it has been split, so the last digit is 1 . The first 4 digits correspond to the four $16 \times 16$ partitions in raster scan order. Here, the first three $0 \mathrm{~s}$ indicate that there is no further split in the first $16 \times 16 \mathrm{CUs}$. The fourth digit in this example is; 1 hence the last $16 \times 16 \mathrm{CU}$ is split into four $8 \times 8$ sub-CUs. And so forth for the rest of the partitions. The total digits needed for a CU are 20. An extra digit is needed to indicate whether the whole $\mathrm{CU}$ is split or not. Thus, 21 digits are used to represent the partitioning of a CU. This splitting approach is chosen due to its simplicity, it is also consistent with the recursive function calls needed to compress a CU in the HM reference software [19].

In this work, modelling the relation between the feature vectors and split decision is performed using C4.5 decision trees and linear classification. The reason for choosing these two classifiers is that decision trees have been used successfully in optimizing HEVC encoders as reported in [20] and linear classifiers have been used successfully in MPEG-2 to HEVC transcoding as reported in [9].

The training of the decision trees uses Kullback-Leibler Divergence (KLD) to choose the best feature variable for each decision branch. The leaves of decision trees represent the CU's split/no split decision. The trained decision trees are then used for predicting the CUs' split decisions. In the multi model solution, all 21 split decisions are predicted individually. Whereas in the three tier solution, the split decision at $64 \times 64$ level is predicted and if it is a split then the $32 \times 32$ split decision is predicted and so forth. The classification accuracy is reported by calculating the percentage of correct split decisions to the total number of instances used for testing.

The multi model linear classifier is used in the same approach, however training is performed using a closedform formula that results in model weights. Split prediction is performed by means of applying the model weights, using dot product, to the feature variables as explained in details in Sect. 4.1.

In the following, we provide a formulation for the multi model classification problem using linear classification.

Denote by $\mathbf{X}=\left[\mathbf{x}_{1}, \mathbf{x}_{2}, \ldots, \mathbf{x}_{n}\right]^{\top}$ is the sequence of MPEG-2 feature vectors where $\mathbf{X} \in \Re^{n x m}$, where $m$ is the dimensionality of the feature vector, and $n$ is the total number of feature vectors. The corresponding CU split decisions are denoted by $\mathbf{S}=\left[\mathbf{s}_{1}, \mathbf{s}_{2}, \ldots, \mathbf{s}_{n}\right]^{T}$ where $\mathbf{S} \in^{n \times L}$, $L$ is the dimensionality of the split decisions, which is 21 , and $n$ is the total number of CUs which is the same as the total number of feature vectors.

In this formalization, the mapping between the feature vectors and the split decisions is performed using a linear classification approach [21]. However, this is not a straightforward process as 21 split decisions need to be classified. As a result, the prediction of split units is formulated as a multi model classification problem.

The training procedure is repeated $L$ times, where $L=21$, to compute $L$ optimum weight vectors $\left\{\mathbf{w}_{i}^{I}\right\}_{I=1 . . L^{\prime}}$ where $\mathbf{w} \in \Re^{m \times 21}$. Each set of weight vectors corresponds to one split decision out of 21 . Recall from Fig. 3 that 21 decisions are required to identify the split structure for a given $\mathrm{CU}$. Since a split decision is binary, two weight vectors are needed for each split decision, $l ; \mathbf{w}_{0}^{l}$ and $\mathbf{w}_{1}^{l}$. Each vector is computed by minimizing the second norm between a linear combination of MPEG-2 train feature vectors $\left(\mathbf{X ~ w}_{j}\right)$ and a HEVC split decision at index / represented by the column vector $\mathbf{y}_{i^{\prime}}^{\prime} \operatorname{argmin}_{w_{i}}\left\|\mathbf{X} \mathbf{w}_{i}-\mathbf{y}_{i}^{\prime}\right\|_{2}$, such that

$\mathbf{w}_{i}^{\prime}=\left(\mathbf{X}^{T} \mathbf{X}\right)^{-1} \mathbf{X}^{T} \mathbf{y}_{i}^{\prime}$

The subscript $i$ is either 0 or 1 corresponding to the 2 classes of split and no split. The $\mathbf{y}_{0}^{\prime}$ is a column vector from the $\mathbf{S}$ matrix at split index $I$, and $\mathbf{y}_{1}^{l}$ is the ones' complement of $\mathbf{y}_{0}^{l}$.

To classify the $L$ split decisions of an incoming MPEG-2 feature vector represented by the row vector $\mathbf{x}_{\mathrm{j}}$, the optimum weights obtained from (1) are used in (2)

$s_{j}^{\prime}=\underset{i}{\operatorname{argmax}}\left(\mathbf{x}_{j} \mathbf{w}_{i}^{\prime}\right)$

This classification process is repeated 21 times $(I=1 . .21)$ for each split decision. The advantage of using this approach for the classification of the 21 split decisions is related to the computation of its model weights. The weights in this solution are calculated using a non-iterative approach as shown in Eq. (1), consequently affecting the speed of the model generation as reported in the experimental results section.

Additionally, a cleanup post-process is needed to make sure that there is no contradiction between the sub-CU and CU-level split flags. That is, if any of the sub-CU split flags is " 1 " then the CU level split flag will be set to "1" as well. For example, if there exists a $32 \times 32$ split flag in $64 \times 64$ CU then the split flag of the later is set to " 1 ". Likewise, if there is a split flag within a sub-CU, then the split flag for that sub-CU is set to " 1 ". For example, if there exists a $16 \times 16$ split flag in $32 \times 32$ sub-CU then the split flag of the later is set to "1".

\subsection{Three tier classification solution}

In this proposed solution, feature variables are extracted from both the MPEG-2 decoder and HEVC re-encoder. Such feature variables result in higher rate-distortion transcoding performance as the HEVC re-encoder features 
are more relevant to the split decisions of the re-encoding part.

During the training phase, feature variables are collected from the MPEG-2 decoder from $4 \times 4 \mathrm{MBs}, 2 \times 2 \mathrm{MBs}$ and $1 \times 1 \mathrm{MBs}$. Since the size of MPEG-2 MBs are $16 \times 16$ pixels, these $M B$ arrangements correspond to $C U$ sizes of $64 \times 64,32 \times 32$ and $16 \times 16$ respectively. Additionally, for the HEVC re-encoder part, a three tier approach is used for feature extraction as follows. For each CU, feature variables are collected at three CU depths; $64 \times 64$, $32 \times 32$ and $16 \times 16$. This results in three feature matrices combining feature variables from both MPEG-2 and HEVC. Each matrix has corresponding split decision flags/ground truth that are recorded during the training phase. Consequently, three classification models are generated at three CU depths. This model generation process is illustrated in Fig. 4.

Having generated the three split classification models, the system can start operating in the transcoding mode.
During which, features are extracted from the MPEG-2 decoder from $4 \times 4,2 \times 2$ and $1 \times 1 \mathrm{MBs}$. The HEVC reencoder starts by extracting features at $64 \times 64$ level only. At this point, a feature vector is formed from the MPEG- $24 \times 4 \mathrm{MBs}$ and the $64 \times 64 \mathrm{CU}$. The $64 \times 64$ classification model is then used to classify this FV as split or no split. If classified as no split, then early CU termination takes place and no further split predictions are performed. Otherwise, if the split decision is predicted as split, then 4 features vectors are created, one for each of the $32 \times 32$ sub-CUs. The $32 \times 32$ classification model is used to predict the split decision of each FV. This process is repeated for the $16 \times 16$ sub-CUs where the $16 \times 16$ classification model can be used to predict their split decisions. This split decision arrangement is illustrated in Fig. 5. It is worth mentioning that early termination algorithms for optimizing HEVC encoders use similar multi-tier arrangements as reported in [20,22].

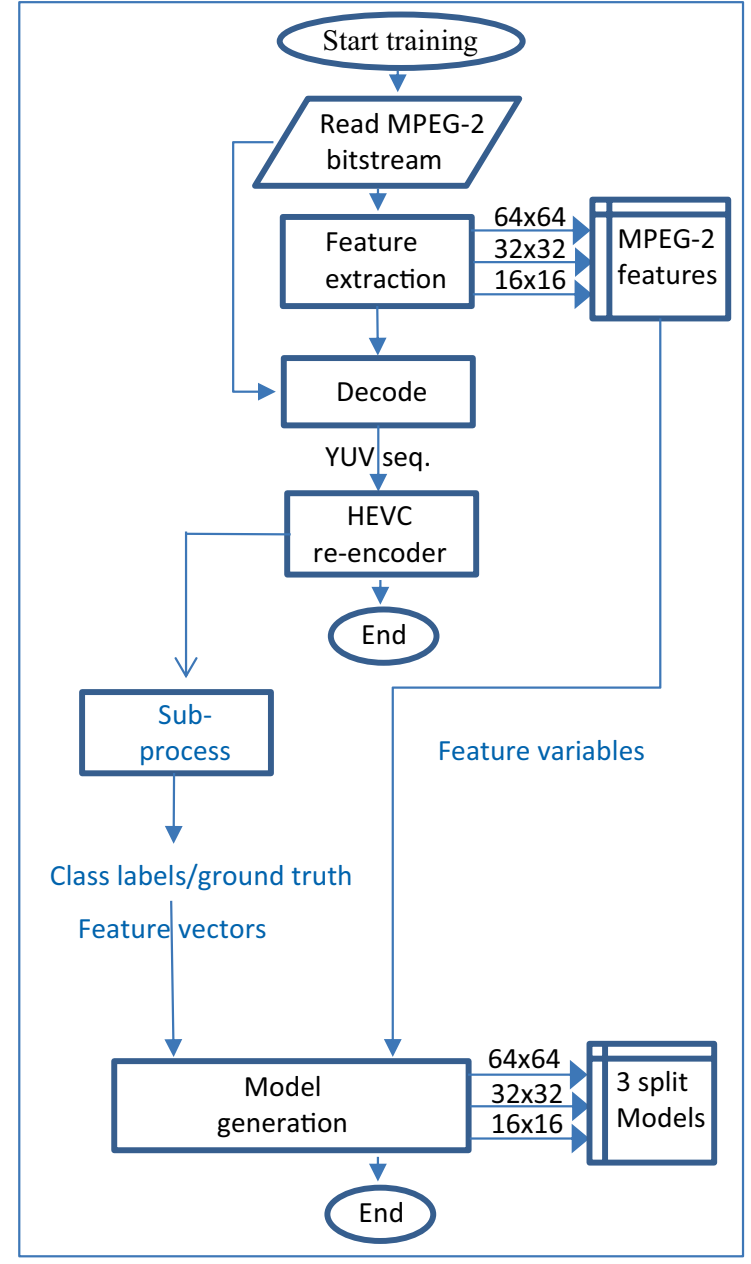

(a) Overview of model generation

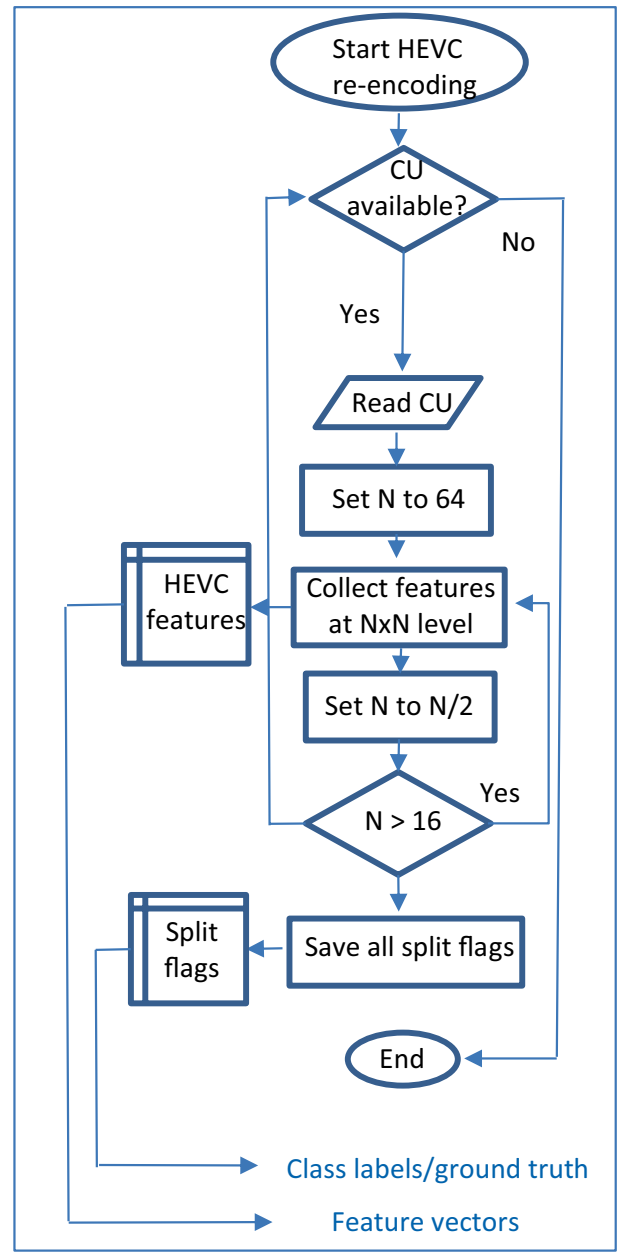

(b) Detailed sub process

Fig. 4 Flowchart of the proposed 3-tier model generation 


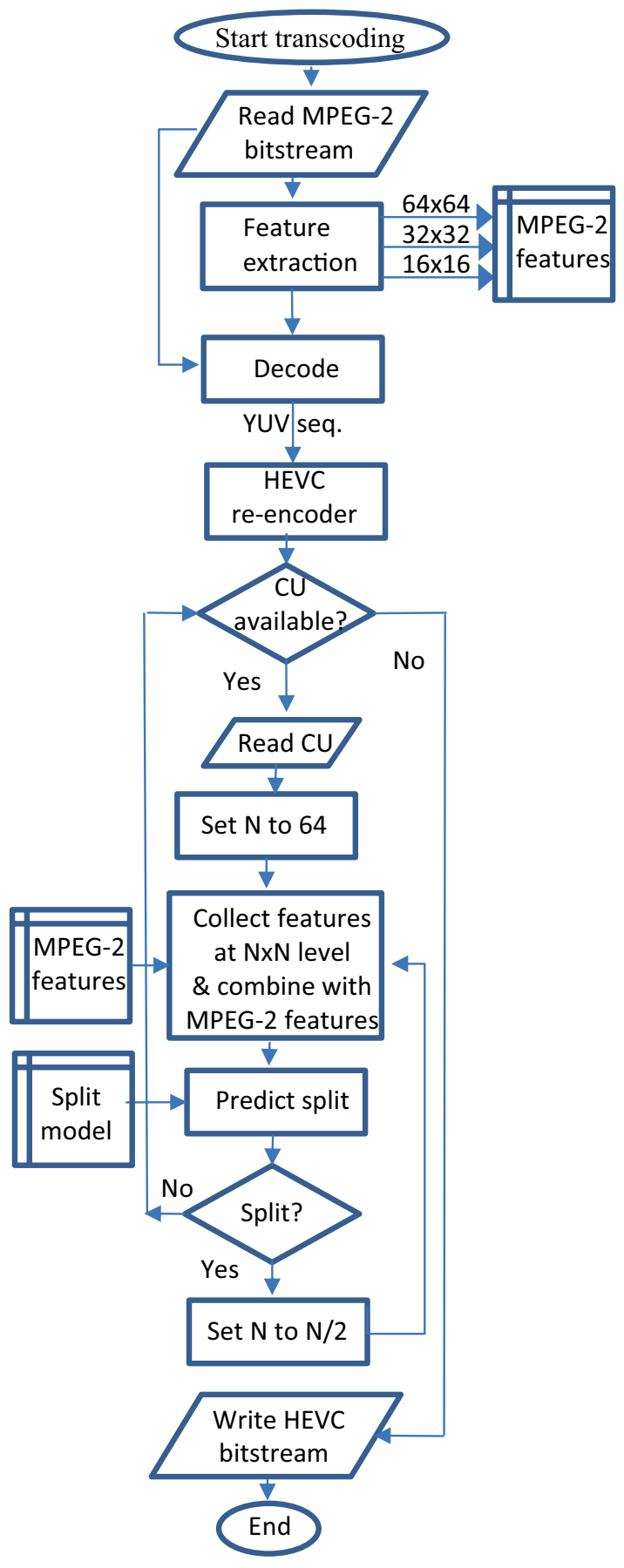

Fig. 5 Flowchart of the proposed split prediction using the 3-tier solution

Similar to the multi model solution, we implement the 3-tier solution using decision trees and linear classifiers. As mentioned previously, the 3-tier solution generates 3 models as opposed to the multi model solution where 21 models are generated. In an attempt to further reduce the model generation time, in this solution we use a reduced set of feature vectors as listed in Table 2 .
These features are collected at 3 tiers pertaining to $64 \times 64,32 \times 32$ and $16 \times 16 \mathrm{CU}$ sizes. The last 12 feature variables are proposed by [20] for the optimization of the HEVC encoder.

\subsection{Summary of proposed solutions}

As mentioned in the introduction, in [9] a MPEG2 to HEVC transcoder was proposed in which one model was generated to predict the depth of one sub CU and replicate it across the whole CU. The model was based on features extracted from the incoming MPEG2 video.

Our proposed solutions are more advanced and more elaborate in terms of feature extraction and model generation. As explained in Sect. 4.1, the first proposed solution is used to predict the splits of the whole coding tree of a given CU and therefore, 21 models are generated instead of one as reported in [9]. As a result, all the 21 split decisions of a CU are predicted which results in a clear performance advantage.

Likewise, as explained in Sect. 4.2, the second proposed solution takes into account the three depth levels of splitting a $\mathrm{CU}$ (i.e. $32 \times 32,16 \times 16$ and $8 \times 8$ ) and therefore, the extracted feature variables are applied to different numbers of incoming MPEG2 MBs pertaining to the three split levels. This results in two major differences with the work reported in [9]. First, three classification models are built; one for each split level. Second, the feature variables are extracted from both MPEG2 and the previously coded CUs in the outgoing HEVC video. As a result, the accuracy of the classification models are boosted as features are extracted from both the incoming MPEG2 video and the outgoing HEVC video.

These major contributions result in significant performance gains in comparison to the work reported in [9] as shown in the experimental results section.

\section{Experimental results}

We use a similar experimental setup to the one reported in [9]. Six test video sequences are used, namely; BasketballDrill $(832 \times 480,50 \mathrm{~Hz})$, PartyScene $(832 \times 480$, $50 \mathrm{~Hz})$, BQMall $(832 \times 480,60 \mathrm{~Hz})$, RaceHorses $(832 \times 480$, $30 \mathrm{~Hz})$, Vidyo $1(1280 \times 720,60 \mathrm{~Hz})$ and BasketballDrive $(1920 \times 1080,50 \mathrm{~Hz})$. The sequences are MPEG-2 encoded using an IPPP... structure.

It is typical in video transcoding to use such a GoP structure without intermediate I or B frames such that picture drift, if any, is magnified. Variable bitrate coding is used with QPs of $\{12,15,20,23\}$ out of 31 . The HEVC coder uses the following corresponding set of QPs $\{25,27,29,30\}$. The 
Table 2 MPEG-2 and HEVC feature variables for 3-tier modeling

\begin{tabular}{|c|c|}
\hline Feature name & Comments \\
\hline MPEG-2 MB skip flag & $\begin{array}{l}\text { Set to } 1 \text { if all } 4 \times 4,2 \times 2 \text { and } 1 \times 1 \text { MBs are skipped. This corresponds to } 64 \times 64 \text {, } \\
32 \times 32 \text { or } 16 \times 16 \text { CUs }\end{array}$ \\
\hline Avg MVs of co-located CU & Average value of MVs of the temporally collocated CU scaled by ref. picture distance \\
\hline PU split mode & Computed prior to splitting a CU \\
\hline \multicolumn{2}{|l|}{ Skip RD cost } \\
\hline \multicolumn{2}{|l|}{$2 \mathrm{~N} \times 2 \mathrm{~N} R D$ cost } \\
\hline \multicolumn{2}{|l|}{$2 \mathrm{~N} \times \mathrm{N} R \mathrm{RD}$ cost } \\
\hline \multicolumn{2}{|l|}{$\mathrm{N} \times 2 \mathrm{~N} R \mathrm{R}$ cost } \\
\hline \multicolumn{2}{|l|}{$\mathrm{N} \times \mathrm{N}$ RD cost } \\
\hline \multicolumn{2}{|l|}{$2 \mathrm{~N} \times \mathrm{uN} R \mathrm{R}$ cost } \\
\hline \multicolumn{2}{|l|}{$2 \mathrm{~N} \times \mathrm{dN} R \mathrm{RD}$ cost } \\
\hline \multicolumn{2}{|l|}{$\mathrm{IN} \times 2 \mathrm{~N} R \mathrm{RD}$ cost } \\
\hline MergeFlag & Binary variables \\
\hline \multicolumn{2}{|l|}{ SkipMergeFlag } \\
\hline AvgDepth & Of surrounding CUs that are previously encoded \\
\hline
\end{tabular}

selected QPs are set such that the reduction in transcoded bitrate is around $50 \%$.

The coding structure is IPPP... using 4 reference frames. The maximum CU size is set to $64 \times 64$. The asymmetric motion partitions tool and the adaptive loop filter tool are both enabled. HEVC reference software HM13.0 is used [19]. In all cases, the HEVC uses the default fast motion estimation (a modified EPZS) and fast mode decision. The first 25 frames of each sequence are used for model generation. The ground truth, which is the true split flags, are computed by encoding the video sequences using the HEVC re-encoder with the same coding parameters explained above. Additionally, for the purpose of comparing the proposed solution against recent state-of-the-art solutions, nine other video sequences are used in this section.

The proposed prediction of split decisions is assessed in the context of video transcoding. This is achieved by comparing the resultant compression results against the brute-force method of full re-encoding. The comparison is performed in terms of excessive bitrate and PSNR. The results of integrating the proposed solutions with a video transcoder are also compared against an existing MPEG-2 to HEVC transcoder of [9].

Lastly, the proposed solutions are assessed in terms of prediction accuracy and computational time.

In the following, the presentation and discussion of the results are divided into 2 subsections, namely; results based on the multi model approach and results based on the 3-tier approach.

\subsection{Multi model approach results}

The percentages of transcoding excessive bitrates based in this solution are reported in Table 3 . The excessive bitrates are computed in comparison to performing full

Table 3 Excessive bitrate (\%) of proposed and reviewed transcoding solutions

\begin{tabular}{|c|c|c|c|c|c|c|c|c|c|c|c|c|}
\hline & \multicolumn{4}{|c|}{ Multi model-Trees } & \multicolumn{4}{|c|}{ Multi model-Linear } & \multicolumn{4}{|c|}{ Reviewed [9] } \\
\hline & \multicolumn{4}{|c|}{$\mathrm{Q}_{\text {in }} \rightarrow \mathrm{Q}_{\text {out }}$} & \multicolumn{4}{|c|}{$\mathrm{Q}_{\text {in }} \rightarrow \mathrm{Q}_{\text {out }}$} & \multicolumn{4}{|c|}{$\mathrm{Q}_{\text {in }} \rightarrow \mathrm{Q}_{\text {out }}$} \\
\hline & $12 \rightarrow 25$ & $15 \rightarrow 27$ & $20 \rightarrow 29$ & $23 \rightarrow 30$ & $12 \rightarrow 25$ & $15 \rightarrow 27$ & $20 \rightarrow 29$ & $23 \rightarrow 30$ & $12 \rightarrow 25$ & $15 \rightarrow 27$ & $20 \rightarrow 29$ & $23 \rightarrow 30$ \\
\hline Race Horses & 1.98 & 2.79 & 3.35 & 3.62 & 1.63 & 2.14 & 2.41 & 2.55 & 4.2 & 5.6 & 6.1 & 6.5 \\
\hline Basket ball Drill & 2.81 & 3.29 & 3.46 & 3.67 & 2.05 & 2.23 & 1.97 & 1.97 & 5.4 & 51 & 4.7 & 4.3 \\
\hline Party Scene & 1.98 & 2.6 & 2.8 & 3.12 & 1.13 & 1.58 & 1.66 & 1.74 & 2.2 & 2.4 & 2.6 & 2.7 \\
\hline BQ-Mall & 3.67 & 4.43 & 4.97 & 5.35 & 2.63 & 3.11 & 3.35 & 3.48 & 5.5 & 6.5 & 6.6 & 6.8 \\
\hline Basket ball Drive & 4.38 & 4.79 & 5.01 & 5.41 & 2.47 & 2.61 & 2.4 & 2.46 & 3.8 & 4.1 & 3.8 & 4 \\
\hline Vidyol & 3.32 & 3.79 & 4.69 & 4.48 & 0.67 & 0.35 & 0.47 & 0.38 & 0.9 & 0.2 & 0.2 & 0 \\
\hline Avg & $3.74 \%$ & & & & $1.98 \%$ & & & & $5.80 \%$ & & & \\
\hline
\end{tabular}


HEVC re-encoding as (transcoding_bitrate - full_re-encoding_bitrate)/full_re-encoding_bitrate* 100 . Each video sequence is transcoded 4 times using the above-mentioned input-output quantization pairs. The percentage of excessive bit rate is reported for each input-output quantization pair. The overall averages are also reported in the table.

In the table, "Multi model-Trees" and "Multi modellinear" refer to the use of decision trees and linear classifiers with the proposed multi model solution. The average excessive bitrates of the four runs show that the proposed solutions, with reference to the reviewed work, are reduced by $36 \%$ and $66 \%$ using decision trees and linear classification respectively. This is a clear transcoding advantage which is due to higher accuracy of predicting split decisions for all sub CUs of a $64 \times 64$ CU. The bitrate results also indicate that the proposed multi model classification approach based on linear classification, results in lower excessive bitrate when compared to multi model classification based on decisions trees. This indicates that there is a linear mapping between the MPEG-2 features and the corresponding split decisions in the HEVC re-encoder.

Moreover, the average PSNR differences in comparison to the re-encoding approach are reported in Table 4.

As the differences are minor, the results in Table 4 are the averages of the four runs per video sequence. The drop in PSNR is computed by subtracting the PSNR of the transcoder from that of the full re-encoder. The results indicate that in comparison to full re-encoding, the PSNR for the proposed solutions are similar. Clearly, a 0.05 or $0.06 \mathrm{~dB}$ difference in PSNR is subjectively negligible.

The prediction accuracy of split decisions plays an important role in transcoding as it affects the accuracy of re-encoding. The prediction accuracy is measured by computing the percentage of correctly predicted split decisions in comparison to full re-encoding. The accuracy is reported in Table 5 for the proposed and reviewed solutions as an average of the four transcoding runs.

Table 4 Average drop in PSNR in $\mathrm{dB}$ of proposed and reviewed transcoding solutions

\begin{tabular}{llll}
\hline & \multicolumn{2}{l}{$\begin{array}{l}\text { Multi } \\
\text { model-Trees }\end{array}$} & $\begin{array}{l}\text { Multi model- } \\
\text { Linear }\end{array}$ \\
\hline RaceHorses & -0.04 & -0.05 & -0.07 \\
BasketballDrill & -0.05 & -0.06 & -0.07 \\
PartyScene & -0.03 & -0.03 & -0.04 \\
BQMall & -0.05 & -0.06 & -0.09 \\
BasketballDrive & -0.05 & -0.06 & -0.06 \\
Vidyo1 & -0.09 & -0.11 & -0.11 \\
Average & -0.05 & -0.06 & -0.08 \\
\hline
\end{tabular}

Table 5 Classification accuracy of CU split decisions

\begin{tabular}{llll}
\hline & $\begin{array}{l}\text { Multi } \\
\text { model-Trees }\end{array}$ & $\begin{array}{l}\text { Multi model_- Reviewed [9] } \\
\text { Linear }\end{array}$ & \\
\hline RaceHorses & $85.3 \%$ & $86.8 \%$ & $59.0 \%$ \\
BasketballDrill & $88.4 \%$ & $90.0 \%$ & $62.0 \%$ \\
PartyScene & $75.1 \%$ & $79.4 \%$ & $58.0 \%$ \\
BQMall & $84.3 \%$ & $87.2 \%$ & $60.0 \%$ \\
BasketballDrive & $88.7 \%$ & $90.4 \%$ & $58.0 \%$ \\
Vidyo1 & $94.7 \%$ & $95.4 \%$ & $73.0 \%$ \\
Average & $86.1 \%$ & $88.2 \%$ & $61.7 \%$ \\
\hline
\end{tabular}

It is shown in the table that both of the proposed solutions have higher prediction accuracy in comparison to the reviewed solution. This is a clear indication that the proposed training approach is more accurate than the existing transcoding solution. The results also indicate that the prediction accuracy using the linear classifier resulted in the highest classification accuracy. This can have a positive influence on reducing the excessive bitrate as reported in Table 4 above.

The classification accuracies of the Vidyo 1 sequence stands out in all solutions. Further investigations into the Vidyo 1 transcoding scenario revealed that the percentage of non-split CUs in the re-encoded video is around $53 \%$. Therefore, it seems that the classification of split decisions in such a sequence is more straightforward.

The performance of the multi-model solution using linear classification generated best results. To further verify the claim that there is a linear mapping between the MPEG-2 features and the corresponding split decisions in the HEVC re-encoder, we repeated the results using the same linear classifier but with second order polynomial expansion of the feature vectors prior to classification. Interestingly, we found that the average classification accuracy of $\mathrm{CU}$ split decisions over 4 transcoding runs dropped to $82.7 \%$ which is inferior to the linear classifier without polynomial expansion. This gives further indication that there is a linear mapping between the feature variables of Table 1 and the CU split decisions.

The proposed solutions work for higher video resolutions as well. Although the highest supported spatial resolution in MPEG-2 is $1920 \times 1080$; nonetheless, the proposed solutions are tested using the old_town_cross sequence which has $3840 \times 2160$ pixels at $50 \mathrm{~Hz}$. In both solutions, the drop in PSNR was trivial; around $0.03 \mathrm{~dB}$, without any excessive bitrate. The prediction accuracy of split decisions was $95.6 \%$.

In summary, based on the results presented in Tables 3, 4 and 5, it is shown that the proposed prediction of CU split decisions using the proposed training solutions are advantageous. The advantage stems 
Table 6 Model generation time in seconds averaged over 4 transcoding runs

\begin{tabular}{lll}
\hline Sequence & Multi model-Trees & $\begin{array}{l}\text { Multi } \\
\text { model- } \\
\text { Linear }\end{array}$ \\
\hline RaceHorses & $2.31 \mathrm{~s}$ & $0.97 \mathrm{~s}$ \\
BasketballDrill & $2.26 \mathrm{~s}$ & $1.28 \mathrm{~s}$ \\
PartyScene & $2.65 \mathrm{~s}$ & $1.25 \mathrm{~s}$ \\
BQMall & $2.12 \mathrm{~s}$ & $1.58 \mathrm{~s}$ \\
Vidyo1 & $6.32 \mathrm{~s}$ & $4.48 \mathrm{~s}$ \\
BasketballDrive & $20.6 \mathrm{~s}$ & $4.53 \mathrm{~s}$ \\
Average & $6.04 \mathrm{~s}$ & $2.35 \mathrm{~s}$ \\
\hline
\end{tabular}

Table 7 Transcoding speedup factors averaged over 4 runs

\begin{tabular}{llll}
\hline Sequence & $\begin{array}{l}\text { Multi model } \\
\text { Trees }\end{array}$ & $\begin{array}{l}\text { Multi model } \\
\text { Linear }\end{array}$ & Reviewed [9] \\
\hline RaceHorses & 1.4 & 1.5 & 2.4 \\
BasketballDrill & 1.7 & 1.8 & 2.5 \\
PartyScene & 1.2 & 1.3 & 2 \\
BQMall & 1.5 & 1.5 & 2.3 \\
BasketballDrive & 2.3 & 2.3 & 2.9 \\
Vidyo1 & 2.6 & 3.0 & 3.1 \\
Average & 1.8 & 1.9 & 2.5 \\
\hline
\end{tabular}

from reducing the excessive bitrate to $1.98 \%$, which is the result of a rather accurate prediction of the $\mathrm{CU}$ split decisions.

In Table 6, we report the model generation time of the proposed solutions. The time computations measured for the experiments in Tables 6 and 7 are carried out on an Intel ${ }^{\circ}$ Core ${ }^{\mathrm{TM}} \mathrm{i} 7 \mathrm{CPU} @ 2.7 \mathrm{GHz}$. The OS is 64-bit Windows 7 Enterprise $\mathrm{N}$ with $16 \mathrm{~GB}$ of memory. As mentioned previously, 21 models are generated, one for each split decision. Each model has a copy of the feature vectors and the corresponding split decision; therefore the models can be generated in parallel as they are independent of each other. In Table 6, the parallel execution time of the models is computed by finding the maximum time required to generate each of the 21 models.

It is shown in the table that the model generation time increases as the spatial resolution of the video increases.

It is also shown that the model generation time using decision trees is higher than that of using linear classifiers. This is an expected result as the latter classifiers use a non-iterative, closed-form solution for the calculation of the model weights. The model generation time of the reviewed work and the multi model solution based on linear classification require the same time as in both solutions the linear classifiers use the same number of feature vectors.
The transcoding speedup factors for the proposed and reviewed solution are reported in Table 7 . The speedup factor is computed by dividing the time required for HEVC full re-encoding by the time required for transcoding with split decision predictions. The speedup factors per sequence are computed for all QP combinations and the average speedup values are reported in Table 7.

Approximately, the speedup factors for the test sequences vary depending on the video content. This is so because the split decisions and the coding tree units of CUs mainly depend on the spatio-temporal content of the video sequence. The time required to compress a sequence is partially related to the complexity of the coding tree units and split decisions. In the transcoding case, coding depths beyond the predicted ones are not tested. It is shown in the table that the average speedup factor for the reviewed solution is higher than that of the proposed solutions. This is expected as the excessive bitrate of the reviewed solution as reported in Table 3 is $5.8 \%$. It is known in video compression that high encoding speedups can be easily achieved at the expense of the rate-distortion performance.

In summary, the proposed multi model solutions are superior to the reviewed transcoding work in terms of reducing the excessive bitrate by up to $66 \%$ as shown in Table 3. PSNR differences on the other hand, are all on average negligible with a slight advantage to the proposed solutions as shown in Table 4. In terms of classification accuracy of split decisions, the proposed solutions achieved around $86 \%$ and $88 \%$ accuracy in comparison to $62 \%$ of the reviewed work as reported in Table 5 . These results justify the noticeable reduction in excessive bitrate that was reported in Table 3. With the 5.8\% excessive bitrate of the reviewed solution, it is no surprise that the transcoding speedup is up to 2.5 in comparison to the proposed solutions that achieved up to 1.9 speedup as reported in Table 7.

Therefore, the proposed solution is superior to the existing work in terms of the prediction accuracy of the CU split decisions and in terms of reducing the excessive transcoding bitrate.

\subsection{Three-tier approach results}

In this sub section, we present and discuss the results based on the three-tier solution using decision trees. The results are compared against the multi model and reviewed work results. We start by presenting the excessive bitrate results as reported in Table 3 above. The results in Table 8 indicate that the proposed 3-tier solution using decision trees is the most accurate as on average, there is no excessive bitrate in comparison to full re-encoding. Recall that in Table 3, the average excessive bitrates for 
Table 8 Excessive bitrate (\%) of proposed 3-tier solution

\begin{tabular}{|c|c|c|c|c|c|c|c|c|}
\hline & \multicolumn{4}{|c|}{ Three-Tier (Trees) } & \multicolumn{4}{|c|}{ Three-Tier (Linear) } \\
\hline & \multicolumn{4}{|c|}{$\mathrm{Q}_{\text {in }} \rightarrow \mathrm{Q}_{\text {out }}$} & \multicolumn{4}{|c|}{$\mathrm{Q}_{\text {in }} \rightarrow \mathrm{Q}_{\text {out }}$} \\
\hline & $12 \rightarrow 25$ & $15 \rightarrow 27$ & $20 \rightarrow 29$ & $23 \rightarrow 30$ & $12 \rightarrow 25$ & $15 \rightarrow 27$ & $20 \rightarrow 29$ & $23 \rightarrow 30$ \\
\hline Race Horses & 0.67 & 0.16 & -0.02 & 0.44 & 0.9 & 0.8 & 2.9 & 3.1 \\
\hline Basket ball Drill & -0.34 & 0 & -0.36 & 0 & 2.3 & 1.5 & 0.8 & 0.6 \\
\hline Party Scene & 0.74 & 0.23 & -0.55 & -0.82 & 10.0 & 7.8 & 4.9 & 4.0 \\
\hline BQ-Mall & 0.67 & -0.06 & -0.26 & -0.26 & 6.7 & 5.2 & 2.6 & 1.7 \\
\hline Vidyol & -0.19 & -0.29 & -0.29 & -0.32 & 8.3 & -0.4 & -0.5 & 0.7 \\
\hline Basketball Drive & -1.43 & -1.11 & -1.37 & -1.59 & -1.6 & -1.3 & -1.6 & -1.3 \\
\hline Avg. all runs & $0.00 \%$ & & & & $2.37 \%$ & & & \\
\hline
\end{tabular}

the two multi model solutions and the reviewed work are $3.74 \%, 1.98 \%$ and $5.8 \%$ respectively. This excellent performance of the 3-tier solution is due to the use of feature variables from both MPEG-2 decoder and HEVC re-encoder part. The feature variables of the HEVC re-encoder are more relevant to the re-encoding part as these variables directly contribute to the split decisions of the HEVC reencoder. It is also shown in the table that the three-tier solution with linear classification results in average excessive bitrate of $2.37 \%$. As mentioned in the previously, the feature variables used in the 3-tier solution are listed in Table 2. These feature variables are different that the ones used for the multi-model solution, which are listed in Table 1. The feature variables in Table 2 are selected based on the fact that in the 3-tier solution, features are selected from the HEVC video whilst encoding the CUs. These features are limited in number and therefore more suitable for decision trees. Further investigation revealed that the classification accuracy of the split decisions at three depth levels is inferior using linear classification in the 3-tier framework.

In some cases, the excessive bitrates reported in Table 8 are negative, which indicates that the transcoder generated slightly lower bitrate than the full re-encoder. This comes at the expense of a slight drop in PSNR as reported in Table 9.

The drop in PSNR is computed by subtracting the PSNR of the transcoder from that of the full re-encoder. Recall that the corresponding averages of the proposed multi model solutions in Table 4 above are -0.05 and $-0.06 \mathrm{~dB}$. The drop in PSNR is slightly higher when the 3-tier framework with linear classifiers is used. Again, this is due to the less accurate $\mathrm{CU}$ split prediction at the three levels of $\mathrm{CU}$ depths as elaborated upon next.

In terms of classification accuracy, the proposed 3-tier solution has a lower classification result in comparison to the multi model approach. The average classification accuracy of the 3-tier solution using decision trees is $81 \%$ in comparison to $86.1 \%$ and $88.2 \%$ of the multi model
Table 9 Average drop in PSNR in dB of proposed and reviewed transcoding solutions

\begin{tabular}{llll}
\hline Sequence & $\begin{array}{l}\text { 3-Tier } \\
\text { Trees }\end{array}$ & $\begin{array}{l}\text { 3-Tier } \\
\text { Linear }\end{array}$ & $\begin{array}{l}\text { Reviewed } \\
{[9]}\end{array}$ \\
\hline RaceHorses & -0.03 & -0.08 & -0.07 \\
BasketballDrill & -0.02 & -0.04 & -0.07 \\
PartyScene & -0.04 & -0.26 & -0.04 \\
BQMall & -0.02 & -0.13 & -0.09 \\
BasketballDrive & -0.01 & -0.03 & -0.06 \\
Vidyo1 & -0.02 & -0.03 & -0.11 \\
Average & -0.02 & -0.096 & -0.08 \\
\hline
\end{tabular}

solutions. This can be justified as follows; in the 3-tier solution, the classifier starts by predicting the split decision for the whole $64 \times 64 \mathrm{CU}$, if the decision is 'no split', then no further split decisions are predicted.

Therefore, if the $64 \times 64$ split decision is incorrect then all of the subsequent $32 \times 32$ and $16 \times 16$ split decisions are incorrect as well. This is not the case for the multi model solutions as the 21 split decisions are predicted separately at the same time. This prediction is followed by a clean-up process in which the $64 \times 64$ split decision can be altered if the $32 \times 32$ or the $16 \times 16$ sub CUs are predicted as being split. Additionally, the average classification accuracies using the 3-tier framework for both linear classification and decisions trees are reported in Table 10.

It is shown in the table that linear classification with the proposed 3-Tier solution that uses the feature variables of Table 2 results in less accurate classification results of CU splits. This justifies the lower performance in terms on excessive transcoding bitrate and drop in PSNR as reported in Tables 8 and 9 above. Further investigation revealed that the average classification accuracy at the first tier $(64 \times 64$ CU level) was $87.6 \%$ using decision trees and $74.3 \%$ using linear classifiers. The classification accuracy of the latter is relatively low. Recall that the $32 \times 32$ split decisions are examined only if the corresponding $64 \times 64$ split 
Table 10 Classification accuracy of CU split using 3-tiers

\begin{tabular}{lll}
\hline Sequence & $\begin{array}{l}3 \text {-Tier } \\
\text { Trees }\end{array}$ & $\begin{array}{l}3 \text {-Tier } \\
\text { Linear }\end{array}$ \\
\hline RaceHorses & $81.6 \%$ & $78.2 \%$ \\
BasketballDrill & $82.4 \%$ & $77.7 \%$ \\
PartyScene & $74.0 \%$ & $63.7 \%$ \\
BQMall & $79.9 \%$ & $74.7 \%$ \\
BasketballDrive & $79.2 \%$ & $75.1 \%$ \\
Vidyo1 & $89.2 \%$ & $83.9 \%$ \\
Average & $81.0 \%$ & $75.5 \%$ \\
\hline
\end{tabular}

decision is predicted as true, therefore, inaccurate prediction of the $64 \times 64$ split decision has an adverse effect on rate-distortion performance.

The model generation time and the transcoding speedup of the proposed 3-tier solution are reported in Table 11.

The average time required for model generation for the proposed multi model solutions are $6.04 \mathrm{~s}$ and $2.35 \mathrm{~s}$ as reported in Table 6 above. In the proposed 3-tier solution, the corresponding average is $3.08 \mathrm{~s}$. Again, the 3-tier solution uses decision trees; hence, the modeling time is expected to be more than the multi model solution based on linear classification. In comparison to the model generation time of the multi model solution using decision tress, the present solution has lower computational complexity as less feature variables are used in this solution. The transcoding speedup on the other hand is lower than that of the multi model solutions. This indicates that the latter solutions predicted a higher percentage of non-splits resulting in faster transcoding and lower video quality.

In summary, the 3-tier solution using decision trees resulted in the best rate-distortion behavior. In terms of model generation time, the 3-tier approach based on linear classification is the fastest, yet this is achieved at the expense of rate-distortion performance.

Additionally, the use of feature variables from both MPEG-2 and HEVC and the prediction of CU splits at

Table 11 Model generation time in seconds and transcoding speedup averaged over 4 runs

\begin{tabular}{lll}
\hline Sequence & Modeling time (s) & Trans. speedup \\
\hline RaceHorses & $2.13 \mathrm{~s}$ & 1.45 \\
BasketballDrill & $1.42 \mathrm{~s}$ & 1.52 \\
PartyScene & $3.61 \mathrm{~s}$ & 1.31 \\
BQMall & $1.53 \mathrm{~s}$ & 1.44 \\
Vidyo1 & $2.79 \mathrm{~s}$ & 2.53 \\
BasketballDrive & $6.97 \mathrm{~s}$ & 1.64 \\
Average & $3.08 \mathrm{~s}$ & 1.65 \\
\hline
\end{tabular}

three different depths resulted in eliminating the excessive transcoding bitrate as shown in Table 8. This is a clear advantage over both, the reviewed work and the proposed multi model solutions that resulted in minimum excessive bit rate of $1.9 \%$ as reported in Table 3. This discussion also holds for the average drop in PSNR as reported in Table 9. This high accuracy in transcoding comes at an expense of lower speedup of 1.65 as reported in Table 5. The speedup of the proposed multi model solution on the other hand was 1.9 as reported in Table 7.

Therefore, the second proposed solution further reduced the transcoding excessive bitrate and enhanced the video quality at the expense of reducing the transcoding speedup. There is a trade-off between transcoding accuracy and transcoding speedup as expected.

Lastly, there are a number of HEVC encoder optimization solutions reported in the literature such as [23-27]. The proposed CU split prediction can be applied to the HEVC encoder and compared to existing literature. For a fair comparison, we apply our 3-tier CU split prediction to the HEVC encoder and assess the accuracy and efficiency using BD-rate [22] and time savings. A lower $B D$-rate value and a higher time saving are desired. In the literature, some researchers use the Computational Complexity Reduction (CCR\%) which is computed as $\left(\right.$ Time $_{\text {reference }}-$ Time $\left._{\text {proposedsolution }}\right) /$ Time $_{\text {reference }} * 100$. Others use $\Delta$ Time (\%) which is defined as $\left(\right.$ Time $_{\text {reference }}-$ Time $\left._{\text {proposedsolution }}\right) /$ Time $_{\text {proposedsolution }}{ }^{*} 100$.

Table 12 lists the video sequences and their resolutions employed for comparison with [23-27] all video sequences are encoded with QPs of $\{22,27,32$, and 37$\}$.

In Table 13, we show the results of predicting the $\mathrm{CU}$ split decisions of HEVC in comparison to [23-27].

In [23], the average BD-rate penalty is 0.62 with a $\Delta$ Time advantage of $53.6 \%$. The proposed solution is faster as the $\Delta$ Time is $82.6 \%$. The time difference is achieved with

Table 12 List of video sequences used

\begin{tabular}{llllll}
\hline Class & ID & Video sequence & Res. & Hz & Bit depth \\
\hline D & D1 & BlowingBubbles & $416 \times 240$ & 50 & 8 \\
& D2 & BQSquare & $416 \times 240$ & 60 & 8 \\
& D3 & BasketballPass & $416 \times 240$ & 50 & 8 \\
C & C1 & ParkScene & $832 \times 480$ & 50 & 8 \\
& C2 & BQMall & $832 \times 480$ & 60 & 8 \\
& C3 & BasketballDrill & $832 \times 480$ & 50 & 8 \\
B & B1 & PartyScene & $1920 \times 1080$ & 24 & 8 \\
& B2 & Cactus & $1920 \times 1080$ & 50 & 8 \\
& B3 & BQTerrace & $1920 \times 1080$ & 60 & 8 \\
A & A1 & Traffic & $2560 \times 1600$ & 30 & 8 \\
& A2 & NebutaFestival & $2560 \times 1600$ & 60 & 10 \\
& A3 & SteamLocomotiveTrain & $2560 \times 1600$ & 60 & 10 \\
\hline
\end{tabular}


Table 13 Accuracy and time savings of prediction CU split decisions

\begin{tabular}{|c|c|c|c|c|c|c|c|c|c|c|c|c|c|}
\hline \multirow[t]{2}{*}{ S. ID } & \multicolumn{3}{|l|}{ Proposed } & \multicolumn{2}{|l|}{ Ref. [23] } & \multicolumn{2}{|l|}{ Ref. [24] } & \multicolumn{2}{|l|}{ Ref. [25] } & \multicolumn{2}{|l|}{ Ref. [26] } & \multicolumn{2}{|l|}{ Ref. [27] } \\
\hline & BD rate $\%$ & CCR\% & $\Delta \mathrm{T} \%$ & $\mathrm{BD}$ rate $\%$ & $\Delta \mathrm{T} \%$ & BD rate $\%$ & CCR\% & BD rate $\%$ & CCR\% & $\mathrm{BD}$ rate $\%$ & CCR\% & $\mathrm{BD}$ rate $\%$ & CCR\% \\
\hline D1 & 1.212 & 31.8 & 52.6 & 0.33 & 54.7 & 1.67 & 25.6 & 0.52 & 36.2 & 1.55 & 42 & 0.9 & 51 \\
\hline $\mathrm{D} 2$ & 0.57 & 36.4 & 68.2 & 0.47 & 56 & 2.34 & 33 & 0.64 & 43.2 & 1.02 & 53 & 0.8 & 46 \\
\hline D3 & 0.72 & 30.1 & 48.4 & 0.65 & 51.2 & 1.17 & 44.5 & 1.02 & 28.4 & 0.98 & 40 & 0.9 & 41 \\
\hline $\mathrm{C} 1$ & 0.67 & 31.8 & 52.2 & 0.25 & 56 & 2.53 & 39.8 & 0.41 & 34.3 & 1.3 & 44 & 1 & 47 \\
\hline $\mathrm{C} 2$ & 0.55 & 31.5 & 50.6 & 0.77 & 50.7 & 1.98 & 42.4 & 0.77 & 40.7 & 1.56 & 43 & 0.9 & 48 \\
\hline $\mathrm{C} 3$ & 0.75 & 36.5 & 63 & 0.52 & 53.3 & 1.72 & 44.2 & 1.06 & 35.2 & 0.95 & 43 & 0.8 & 45 \\
\hline B1 & 0.35 & 43.6 & 92.3 & 0.49 & 50.3 & 1.54 & 30.3 & 0.4 & 41.6 & 1.3 & 44 & 0.8 & 52 \\
\hline B2 & 0.51 & 43.5 & 88.8 & 0.57 & 48 & 2.48 & 43.8 & 0.48 & 42.7 & 1.81 & 51 & 1.1 & 49 \\
\hline B3 & 0.56 & 45.9 & 114.2 & 0.87 & 59.9 & 1.62 & 31.9 & 1.13 & 44 & 1.7 & 57 & 1 & 52 \\
\hline $\mathrm{A} 1$ & 0.6 & 48 & 113.8 & 0.71 & 55.2 & 3.25 & 49.7 & 0.57 & 43.6 & 1.86 & 59 & 0.9 & 53 \\
\hline $\mathrm{A} 2$ & 0.31 & 48.6 & 110.5 & 0.78 & 58.8 & 2.18 & 37.9 & 1.01 & 47.6 & - & - & - & - \\
\hline A3 & -0.34 & 52.4 & 136.9 & 0.98 & 48.7 & 1.99 & 30.9 & 1.43 & 22.1 & - & - & - & - \\
\hline Avg & 0.54 & 40 & 82.62 & 0.62 & 53.57 & 2.04 & 37.8 & 0.79 & 38.3 & 1.4 & 47.6 & 0.91 & 48.4 \\
\hline
\end{tabular}

an enhanced BD-rate of 0.54 instead of 0.62. In [24], the average BD-rate penalty was 2.0 with a CCR of $37.8 \%$. In this case, the proposed solution is more accurate as the $\mathrm{BD}$-rate is noticeably lower. The proposed solution is also faster as the CCR is $40 \%$ versus $37.8 \%$ for the proposed solution.

In [25], the average BD-rate penalty is 0.79 which is inferior to the proposed solution and the CCR \% saving is lower as well. In [26, 27], the CCR are $47.6 \%$ and $48.4 \%$, however, the computational savings come at a relatively high BD-rate cost of 0.91 and 1.4 respectively.

Lastly, the work reported in [28] proposed CU split prediction based on the motion features and rate-distortion cost of the $\mathrm{N} \times \mathrm{N}$ inter mode. A MV reuse scheme is also proposed to expedite compression. The CCR\% results reported range from 55 to $61 \%$. However, this speedup comes at the expense of high BD-rate loss of 1.93-2.33.

In conclusion, applying the proposed solution to the HEVC encoder results in high time savings whilst enhancing the compression efficiency. Incorporating the proposed solution in the MPEG-2 to HEVC transcoder, as described in Sect. 4, resulted in an efficient video transcoder as evident in the transcoding results of Sects. 5.1 and 5.2 above.

\section{Conclusion}

Two novel solutions for transcoding MPEG-2 video into HEVC are proposed where split decisions of HEVC CUs are represented as a sequence of 21 binary numbers. Consequently, the split decisions of a CU are predicted using 21 classification models. Alternatively, the split decisions are grouped into 3 depth levels and consequently,
3 classification models are generated. The two solutions were referred to as multi models and three-tier solutions respectively. In both solutions, the transcoder starts by operating in training mode in which feature vectors are mapped to split decisions. Once the classification models are generated, the system switches to transcoding mode.

It was shown in the experimental results that the 3-tier transcoding solution generates rate-distortion results that are very close to those of the full re-encoding counterpart. In fact, it was shown that the excessive bitrate can on average be eliminated in comparison to full re-encoding. Surely, this increase in transcoding accuracy comes at the expense of speedup when compared to other transcoding solutions. It was also shown that, depending on the classifier used, the model generation time varies from 2.35 to $6.04 \mathrm{~s}$ and 0.08 to $3.1 \mathrm{~s}$ for the multi model and 3-tier solutions respectively.

\section{Compliance with ethical standards}

Conflict of interest All authors declare that they have no conflict of interest.

\section{References}

1. ISO/IEC 23008-2:2015 (2015) Information technology-High efficiency coding and media delivery in heterogeneous environments-Part 2: High efficiency video coding, ISO/IEC Standard

2. Shanableh T, Ghanbari M (2000) Heterogeneous video transcoding into lower spatio temporal resolutions with different encoding formats. IEEE Trans Multimed 2(2):101-111

3. Shanableh T, Ghanbari M (2003) Hybrid DCT/pixel domain architecture for heterogeneous video transcoding. Sig Process Image Commun 18(8):601-620 
4. Peixoto E, Shanableh T, Izquierdo E (2014) H264/AVC to HEVC video transcoder based on dynamic thresholding and content modeling. IEEE Trans Circuits Syst Video Technol 24(1):99-112

5. Peixoto E, Macchiavello B, Mintsu Hung E, Shanableh T, Izquierdo E (2013) An H.264/Avc to Hevc video transcoder based on mode mapping. In: IEEE international conference on image processing (ICIP), Melbourne, Australia

6. Chen Y, Wen Z, Wen J, Tang M, Tao P (2015) Efficient software H.264/AVC to HEVC transcoding on distributed multicore processors. IEEE Trans Circuits Syst Video Technol 25(8):1423-1434

7. Jiang Wei, Chen Yaowu, Tian Xiang (2014) Fast transcoding from H.264 to HEVC based on region feature analysis. Multimed Tools Appl 73(3):2179-2200

8. Jiang W, Chenc YW (2013) Low-complexity transcoding from H.264 to HEVC based on motion vector clustering. Electron Lett 49(19):1224-1226

9. Shanableh T, Peixoto E, Izquierdo E (2013) MPEG-2 to HEVC video transcoding with content-based modeling. IEEE Trans Circuits Syst Video Technol 23(7):1191-1196

10. Chen Y, Zhou Y, Wen J (2016) Efficient software HEVC to AVS2 transcoding. Information 7(3):53

11. De La Torre E, Rodriguez-Sanchez R, Martínez JL (2015) Fast video transcoding from HEVC to VP9. IEEE Trans Consum Electron 61(3):336-343

12. Van Pham L, De Praeter J, Van Wallendael G, Van Leuven S, De Cock J, Van De Walle R (2016) Efficient bit rate transcoding for high efficiency video coding. IEEE Trans Multimed 18(3):364-378

13. Wang J, Li L, Zhi G, Zhang Z, Zhang H (2017) Efficient algorithms for HEVC bitrate transcoding. Multimed Tools Appl 76(24):26581-26601

14. Lin C-S, Yang W-J, Su C-W (2016) FITD: Fast Intra Transcoding from H.264/AVC to high efficiency video coding based on DCT coefficients and prediction modes. J Vis Commun Image Represent 38:130-140

15. Diaz-Honrubia AJ, Martinez JL, Cuenca $P$ (2018) A fast intra H.264/AVC to HEVC transcoding system. Multimed Tools Appl 77(5):6367-6384

16. Díaz-Honrubia A, Cebrián-Márquez G, Martínez J, Cuenca P, Puerta J, Gámez J (2016) Low-complexity heterogeneous architecture for H.264/HEVC video transcoding. J Real Time Image Proc 12(2):311-327

17. Liu MF, Zhong G-Y, He X-H, Qing L-B (2016) Transcoding method from H264/AVC to high efficiency video coding based on similarity of intraprediction, interprediction, and motion vector. J Electron Imaging 25(5):053028

18. Mora E, Cagnazzo M, Dufaux F (2017) AVC to HEVC transcoder based on quadtree limitation. Multimed Tools Appl 76(6):8991-9015

19. Kim I-K, McCann KD, Sugimoto K, Bross B, Han W-J, Sullivan GJ (2013) High efficiency video coding (HEVC) test model 13 (HM13) encoder description. In: JCT-VC O1002, 15th meeting of Joint Collaborative Team on Video Coding of ITU-T SG 16 WP 3 and ISO/IEC JTC 1

20. Correa G, Assuncao PA, Agostini LV, da Silva Cruz LA (2015) Fast HEVC encoding decisions using data mining. IEEE Trans Circuits Syst Video Technol 25(4):660-673

21. Shanableh T, Assaleh K (2010) Feature modeling using polynomial classifiers and stepwise regression. Neurocomputing 73(10-12):1752-1759

22. Bjøntegaard G (2008) Improvements of the BD-PSNR model, document VCEG-Al11, ITU-T SG16/Q6, Berlin, Germany

23. Kim H, Park R (2016) Fast CU partitioning algorithm for HEVC using an online-learning-based Bayesian decision rule. IEEE Trans Circuits Syst Video Technol 26(1):130-138

24. Xiong J, Li H, Wu Q, Meng F (2014) A fast HEVC inter CU selection method based on pyramid motion divergence. IEEE Trans Multimed 16(2):559-564

25. Yoo H, Suh J (2014) Fast coding unit decision based on skipping of inter and intra prediction units. Electron Lett 50(10):750-752

26. Tai K-H, Hsieh M-Y, Chen M-J, Chen C-Y, Yeh C-H (2017) A fast HEVC encoding method using depth information of collocated CUs and RD cost characteristics of PU modes. IEEE Trans Broadcast 63(4):680-692

27. Tang M, Chen X, Gu J, Han Y, Wen J, Yang S (2018) Accelerating HEVC encoding using early-split. IEEE Signal Process Lett 25(2):209-213

28. Mallikarachchi T, Talagala D, Arachchi H, Fernando A (2016) Content-adaptive feature-based CU size prediction for fast low-delay video encoding in HEVC. IEEE Trans Circuits Syst Video Technol 28(3):693-705

Publisher's Note Springer Nature remains neutral with regard to jurisdictional claims in published maps and institutional affiliations. 